\title{
Catalytic Conversion of a Chitin-Derived Sugar Alcohol to an Amide-Containing Isosorbide Analog
}

Takuya Sagawa ${ }^{\dagger}$, Hirokazu Kobayashi*广, Chinatsu Murata ${ }^{\ddagger}$,Yukatsu Shichibu ${ }^{\ddagger}$, Katsuaki Konishi*, Atsushi Fukuoka*广

$\uparrow$ Institute for Catalysis, Hokkaido University, Kita 21 Nishi 10, Kita-ku, Sapporo, Hokkaido 001-0021, Japan.

\$ Graduate School of Environmental Science, Hokkaido University, Hokkaido

University, Kita 10 Nishi 5, Kita-ku, Sapporo, Hokkaido 060-0810, Japan.

E-mail: kobayashi.hi@cat.hokudai.ac.jp (H.K.); fukuoka@cat.hokudai.ac.jp (A.F.)

-Totals- 13 pages, 2 notes, 2 schemes, 10 figures, 1 table

Table of Contents

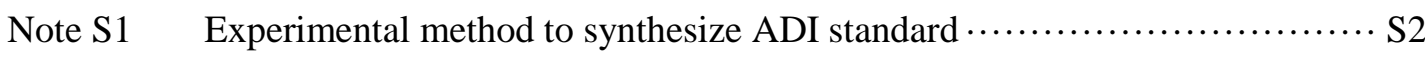

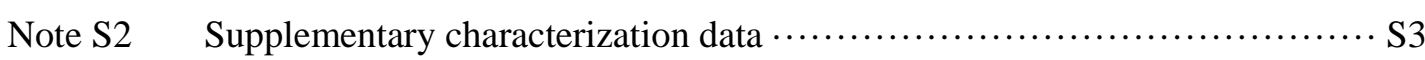

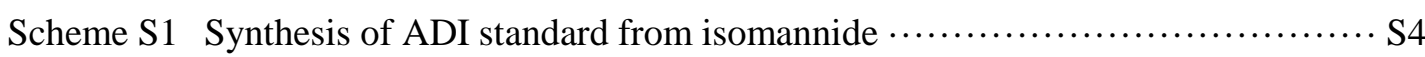

Scheme $\mathrm{S} 2$ 1,4-Dehydration of ADS by (A) $\mathrm{S}_{\mathrm{N}} 1$ reaction at $\mathrm{C} 4$ center,

(B) $\mathrm{S}_{\mathrm{N}} 2$ reaction at $\mathrm{C} 4$ center, and $(\mathrm{C}) \mathrm{S}_{\mathrm{N}} 1$ reaction at $\mathrm{C} 1$ center $\cdots \cdots \cdots \cdots \cdots \cdot \mathrm{S} 5$

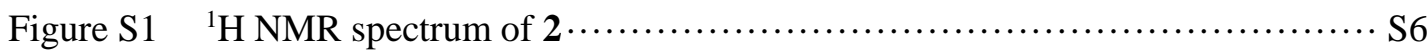

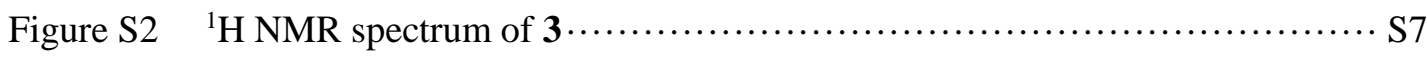

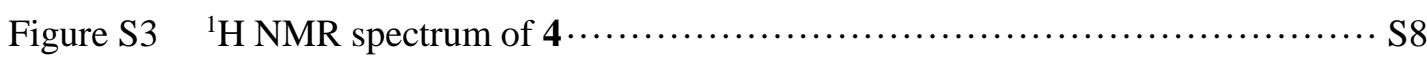

Figure S4 $\quad{ }^{1} \mathrm{H}$ NMR spectra of the standard ADI synthesized from isomannide

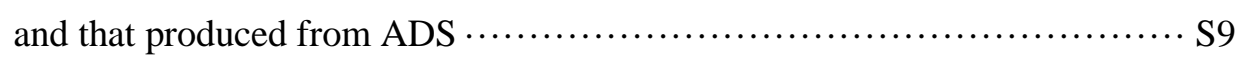

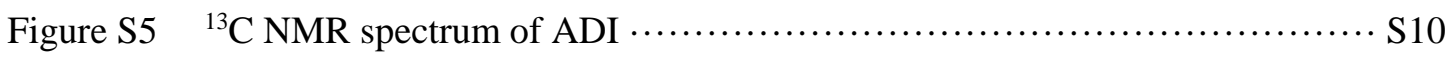

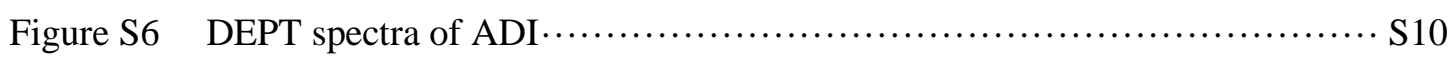

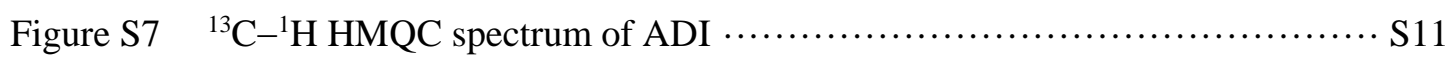

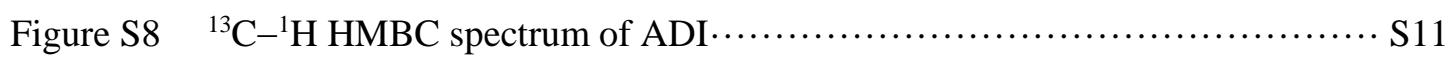

Figure S9 LR-APCI-MS of ADI produced form ADS $\ldots \ldots \ldots \ldots \ldots \ldots \ldots \ldots \ldots \ldots \ldots \ldots \ldots \ldots \ldots \ldots \ldots$

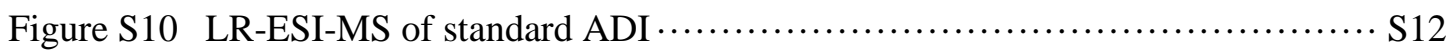

Table S1 Dehydration cyclization of ADS and sorbitol by $\mathrm{H}_{2} \mathrm{SO}_{4} \ldots \ldots \ldots \ldots \ldots \ldots \ldots$ S13 


\section{Note S1: Experimental method to synthesize ADI standard}

All reactions were performed under an Ar atmosphere. First, the hydroxyl groups of isomannide were protected by tert-butyldimethylsilyl groups. Isomannide (1, $1.46 \mathrm{~g}$, $10.0 \mathrm{mmol})$ and imidazole $(0.681 \mathrm{~g}, 10.0 \mathrm{mmol})$ were dissolved in dried DMF (10 mL), and tert-butyldimethylchlorosilane $(1.66 \mathrm{~g}, 11.0 \mathrm{mmol})$ was added to the mixture. The mixture was stirred at room temperature overnight, and then the products were extracted with $\mathrm{Et}_{2} \mathrm{O}$. Volatile compounds were removed under reduced pressure $(10 \mathrm{kPa})$. The solid residue was purified by silica gel column chromatography with EtOAc/hexane $(2 / 3 \mathrm{vol} / \mathrm{vol})$ as an eluent. Compound 2 was collected as the first fraction $(0.600 \mathrm{~g}, 1.60$ mmol, 16\%; colorless liquid; $\mathrm{Rf}=0.87$ ), and compound 3 was obtained as the second fraction (0.765 g, $2.94 \mathrm{mmol}, 29 \%$; white solid; Rf=0.38). 2: ${ }^{1} \mathrm{H}$ NMR (600 MHz, $\left.\mathrm{CDCl}_{3}\right): \delta 0.09\left(\mathrm{~s}, 6 \mathrm{H}, \mathrm{CH}_{3}\right), 0.11\left(\mathrm{~s}, 12 \mathrm{H}, \mathrm{CH}_{3}\right), 0.91\left(\mathrm{~s}, 12 \mathrm{H}, \mathrm{CH}_{3}\right), 3.60(\mathrm{t}, J=7.8 \mathrm{~Hz}$, 2H), $3.86(\mathrm{t}, J=6.6 \mathrm{~Hz}, 1 \mathrm{H}), 4.31(\mathrm{~m}, 4 \mathrm{H})$ (Figure S1). 3: ${ }^{1} \mathrm{H} \mathrm{NMR}\left(600 \mathrm{MHz}, \mathrm{CDCl}_{3}\right)$ : $\delta 0.11\left(\mathrm{~s}, 3 \mathrm{H}, \mathrm{CH}_{3}\right), 0.13\left(\mathrm{~s}, 3 \mathrm{H}, \mathrm{CH}_{3}\right), 0.91\left(\mathrm{~s}, 9 \mathrm{H}, \mathrm{CH}_{3}\right), 3.17(\mathrm{~d}, J=8.9 \mathrm{~Hz}, 1 \mathrm{H}), 3.73$ (m, 2H), $3.93(\mathrm{~m}, 2 \mathrm{H}), 4.18(\mathrm{dt}, J=3.9,5.5 \mathrm{~Hz}, 1 \mathrm{H}), 4.25(\mathrm{~d}, J=4.8,5.5 \mathrm{~Hz}, 1 \mathrm{H}), 4.38$ (t, $J=5.5 \mathrm{~Hz}, 1 \mathrm{H}), 4.50(\mathrm{~d}, J=5.5 \mathrm{~Hz}, 1 \mathrm{H})$ (Figure $\mathrm{S} 2)$.

Second, the hydroxyl group of $\mathbf{3}$ was modified with a trifluoromethanesulfonyl (Tf) group. Compound $3(0.521 \mathrm{~g}, 2.00 \mathrm{mmol})$ and pyridine $(0.193 \mathrm{~mL}, 2.39 \mathrm{mmol})$ were dissolved in dried $\mathrm{CH}_{2} \mathrm{Cl}_{2}$. After the mixture was cooled to $0{ }^{\circ} \mathrm{C}$, trifluoromethanesulfonic anhydride $(0.402 \mathrm{~mL}, 2.39 \mathrm{mmol})$ was added. The mixture was stirred at room temperature overnight, and then the reaction mixture was washed successively with $1 \mathrm{M} \mathrm{HCl}$ aq. $(20 \mathrm{~mL}), \mathrm{H}_{2} \mathrm{O}(20 \mathrm{~mL})$, saturated $\mathrm{NaHCO}_{3}$ aq. $(20 \mathrm{~mL})$, and saturated brine. Volatile compounds in the organic layer were removed under reduced pressure $(10 \mathrm{kPa}$, and then less than $0.1 \mathrm{kPa})$, and 4 was obtained in $62 \%$ yield $(0.490 \mathrm{mg})$ as a pale brown solid. 4: ${ }^{1} \mathrm{H} \mathrm{NMR}\left(600 \mathrm{MHz}, \mathrm{CDCl}_{3}\right): \delta 0.11\left(\mathrm{~s}, 3 \mathrm{H}, \mathrm{CH}_{3}\right)$, $0.12\left(\mathrm{~s}, 3 \mathrm{H}, \mathrm{CH}_{3}\right), 0.91(\mathrm{~s}, 9 \mathrm{H}, t-\mathrm{Bu}), 3.66(\mathrm{t}, J=8.2 \mathrm{~Hz}, 1 \mathrm{H}), 3.96(\mathrm{~m}, 2 \mathrm{H}), 4.13(\mathrm{~m}$, 1H), $4.32(\mathrm{~m}, 2 \mathrm{H}), 4.69$ (t, $J=5.5 \mathrm{~Hz}, 1 \mathrm{H}), 5.17(\mathrm{~m}, 1 \mathrm{H})$ (Figure S3).

Third, we conducted a four-step reaction sequence to obtain ADI from 4 . Compound 4 $(0.196 \mathrm{~g}, 0.50 \mathrm{mmol})$ was dissolved in dry DMF $(10 \mathrm{~mL})$. After the solution was cooled to $0{ }^{\circ} \mathrm{C}, \mathrm{NaN}_{3}(0.130 \mathrm{mg}, 2.00 \mathrm{mmol})$ was added. The mixture was stirred at room temperature for $1 \mathrm{~h}$, and the product was extracted into the EtOAc $(10 \mathrm{~mL})$ layer in the presence of $\mathrm{H}_{2} \mathrm{O}(10 \mathrm{~mL})$. Volatile compounds in the organic layer were removed under reduced pressure (less than $0.1 \mathrm{kPa}$ ), and 5 was obtained as a pale yellow liquid. Compound 5, $\mathrm{H}_{2} \mathrm{O}(0.500 \mathrm{~mL})$, and $\mathrm{PPh}_{3}(0.200 \mathrm{~g}, 0.763 \mathrm{mmol})$ were dissolved in dry $\mathrm{THF}$, and then the solution was refluxed for $3 \mathrm{~h}$. After the mixture was cooled to room 
temperature, EtOAc $(10 \mathrm{~mL})$ and $\mathrm{CaCl}_{2}$ were added. The mixture was filtered, and the volatile compounds in the filtrate were evaporated. This method gave $\mathbf{6}$ as a pale yellow liquid. Compound 6 and $\mathrm{Ac}_{2} \mathrm{O}(0.110 \mathrm{~mL}, 1.00 \mathrm{~mol})$ were dissolved in $\mathrm{MeOH}(5 \mathrm{~mL})$, and the solution was stirred at room temperature overnight. The products were extracted by EtOAc $(10 \mathrm{~mL})$. Volatile compounds in the organic layer were removed under reduced pressure (less than $0.1 \mathrm{kPa}$ ), and 7 was obtained as a colorless liquid. Compound 7 and tetrabutylammonium fluoride $(0.209 \mathrm{~g}, 0.800 \mathrm{~mol})$ were dissolved in dry THF $(2.0 \mathrm{~mL})$ and $\mathrm{H}_{2} \mathrm{O}(0.800 \mathrm{~mL})$, and the solution was stirred at room temperature for $8 \mathrm{~h}$. The volatile compounds were removed under reduced pressure (less than $0.1 \mathrm{kPa}$ ). The solid residue was purified by silica gel column chromatography with $\mathrm{MeOH} / \mathrm{CHCl}_{3}(1 / 9 \mathrm{vol} / \mathrm{vol})$ as an eluent. ADI was collected as the second fraction (0.028 g, $0.148 \mathrm{mmol}, 30 \%$; white solid; $\mathrm{Rf}=0.27)$.

\section{Note S2: Supplementary characterization data}

1,4-Anhydro-ADS: ${ }^{1} \mathrm{H}$ NMR (600 MHz, $\left.\mathrm{D}_{2} \mathrm{O}\right): \delta 1.97\left(\mathrm{~s}, 3 \mathrm{H}, \mathrm{CH}_{3}\right), 3.62(\mathrm{~d}, J=5.5$, $\left.12.4 \mathrm{~Hz}, 1 \mathrm{H}, \mathrm{CH}_{2}\right), 3.70\left(\mathrm{~d}, J=8.9 \mathrm{~Hz}, 1 \mathrm{H}, \mathrm{CH}_{2}\right), 3.77-3.84(\mathrm{~m}, 2 \mathrm{H}), 3.87-3.91(\mathrm{~m}, 1 \mathrm{H}$, $\mathrm{CH}), 4.20-4.27(\mathrm{~m}, 3 \mathrm{H}) .{ }^{13} \mathrm{C}$ NMR (151 MHz, $\left.\mathrm{D}_{2} \mathrm{O}\right): \delta 24.9,60.7,61.6,66.6,72.0,73.9$, 78.1, 83.2, 177.1. LRMS (LR-ESI-TOF) calcd. for $\mathrm{C}_{8} \mathrm{H}_{16} \mathrm{NO}_{5} 206[\mathrm{M}+\mathrm{H}]^{+}$; found 206.

3,6-Anhydro-ADS: ${ }^{1} \mathrm{H}$ NMR (600 MHz, $\left.\mathrm{D}_{2} \mathrm{O}\right): \delta 2.02\left(\mathrm{~s}, 3 \mathrm{H}, \mathrm{CH}_{3}\right), 3.63(\mathrm{dd}, J=7.2$, $10.0 \mathrm{~Hz}, 1 \mathrm{H}), 3.80-3.88(\mathrm{~m}, 2 \mathrm{H}), 3.98-4.06(\mathrm{~m}, 2 \mathrm{H}), 4.11(\mathrm{t}, J=4.8 \mathrm{~Hz}, 1 \mathrm{H}, \mathrm{CH})$, $4.20-4.28(\mathrm{~m}, 1 \mathrm{H}, \mathrm{CH}) .{ }^{13} \mathrm{C} \mathrm{NMR}\left(151 \mathrm{MHz}, \mathrm{D}_{2} \mathrm{O}\right): \delta 25.5,55.8,63.7,73.8,74.5,76.1$, 83.0, 176.4. LRMS (LR-ESI-TOF) calcd. for $\mathrm{C}_{8} \mathrm{H}_{16} \mathrm{NO}_{5} 206[\mathrm{M}+\mathrm{H}]^{+}$; found 206.

1,5-Anhydro-ADS: ${ }^{1} \mathrm{H}$ NMR (600 MHz, $\left.\mathrm{D}_{2} \mathrm{O}\right): \delta 2.00\left(\mathrm{~s}, 3 \mathrm{H}, \mathrm{CH}_{3}\right), 3.24(\mathrm{t}, J=11.0 \mathrm{~Hz}$, $1 \mathrm{H}, \mathrm{CH}), 3.31-3.44(\mathrm{~m}, 2 \mathrm{H}), 3.51(\mathrm{t}, J=11.3 \mathrm{~Hz}, 1 \mathrm{H}, \mathrm{CH}), 3.65(\mathrm{dd}, J=5.7,11.3 \mathrm{~Hz}$, $\left.1 \mathrm{H}, \mathrm{CH}_{2}\right), 3.80-3.95(\mathrm{~m}, 3 \mathrm{H}) .{ }^{13} \mathrm{C}$ NMR (151 MHz, $\left.\mathrm{D}_{2} \mathrm{O}\right): \delta 24.7,54.0,63.6,69.8,72.9$, 77.5, 80.1, 177.4. LRMS (LR-ESI-TOF) calcd. for $\mathrm{C}_{8} \mathrm{H}_{16} \mathrm{NO}_{5} 206[\mathrm{M}+\mathrm{H}]^{+}$; found 206.

ADI: ${ }^{1} \mathrm{H}$ NMR $\left(600 \mathrm{MHz}, \mathrm{CDCl}_{3}\right): \delta 1.99\left(\mathrm{~s}, 3 \mathrm{H}, \mathrm{CH}_{3}\right), 2.59(\mathrm{~s}, 1 \mathrm{H}, \mathrm{OH}), 3.62(\mathrm{dd}, J=$ 5.5, 9.7 Hz, 1H, $\left.\mathrm{CH}_{2}\right), 3.86\left(\mathrm{dd}, J=5.5,9.7 \mathrm{~Hz}, 1 \mathrm{H}, \mathrm{CH}_{2}\right), 3.89(\mathrm{~d}, J=9.6 \mathrm{~Hz}, 1 \mathrm{H}$, $\left.\mathrm{CH}_{2}\right), 3.99\left(\mathrm{dd}, J=4.1,9.6 \mathrm{~Hz}, 1 \mathrm{H}, \mathrm{CH}_{2}\right), 4.30(\mathrm{~m}, 1 \mathrm{H}, \mathrm{CH}), 4.41(\mathrm{~d}, J=4.1 \mathrm{~Hz}, 1 \mathrm{H}$, $\mathrm{CH}), 4.48(\mathrm{dd}, J=4.8,5.9 \mathrm{~Hz}, 1 \mathrm{H}, \mathrm{CH}), 4.55(\mathrm{t}, J=4.8 \mathrm{~Hz}, 1 \mathrm{H}, \mathrm{CH}), 5.71(\mathrm{~s}, 1 \mathrm{H}, \mathrm{NH})$ (Figure S4). ${ }^{13} \mathrm{C}$ NMR (151 MHz, $\left.\mathrm{CDCl}_{3}\right): \delta 23.3,56.9,72.2,73.6,74.1,81.7,86.7$, 169.8 (Figure S5). HRMS (HR-APCI-TOF) calcd. for $\mathrm{C}_{8} \mathrm{H}_{14} \mathrm{NO}_{4} 188.09173[\mathrm{M}+\mathrm{H}]^{+}$; found 188.09196 . 
For the assignment of NMR peaks to determine the structures of these compounds, we measured distortional enhancement by polarization transfer (DEPT), ${ }^{13} \mathrm{C}-{ }^{1} \mathrm{H}$ heteronuclear multiple quantum coherence (HMQC), and ${ }^{13} \mathrm{C}-{ }^{1} \mathrm{H}$ heteronuclear multiple bond correlation (HMBC) spectra (data not shown except for ADI).
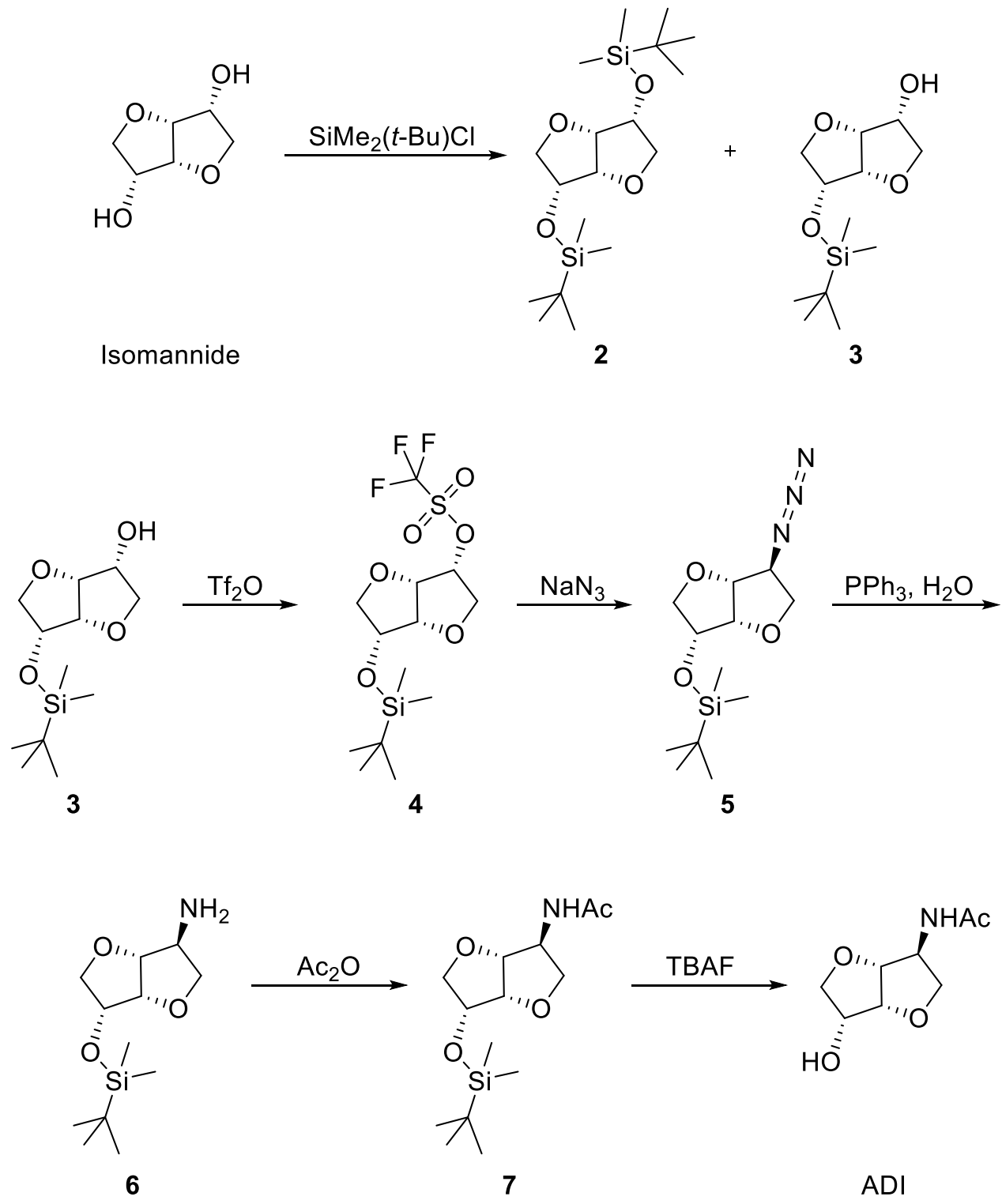

Scheme S1. Synthesis of ADI standard from isomannide. 
(A) $S_{N} 1$ reaction at $C 4$

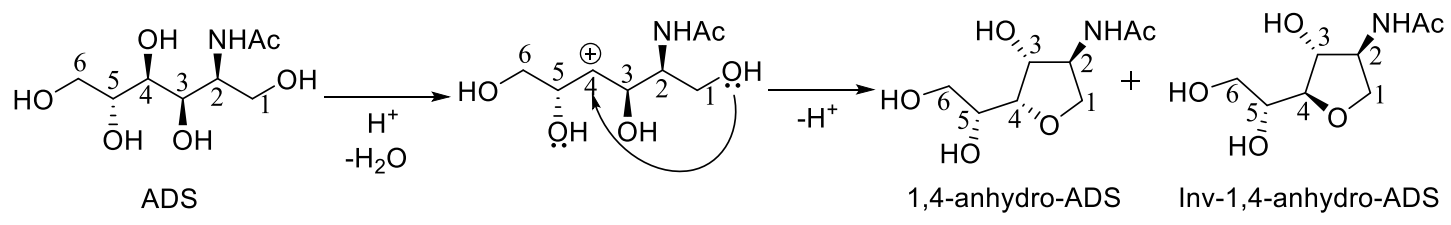

(B) $S_{N} 2$ reaction at $C 4$

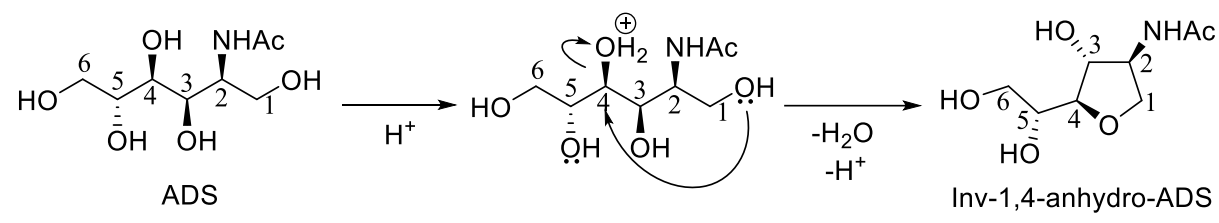

(C) $S_{N} 1$ reaction at $C 1$

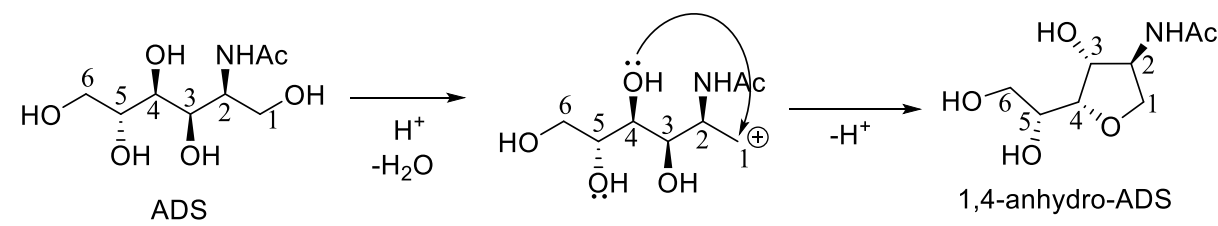

Scheme S2. 1,4-Dehydration of ADS by (A) $\mathrm{S}_{\mathrm{N}} 1$ reaction at $\mathrm{C} 4$ center, (B) $\mathrm{S}_{\mathrm{N}} 2$ reaction at $\mathrm{C} 4$ center, and (C) $\mathrm{S}_{\mathrm{N}} 1$ reaction at $\mathrm{C} 1$ center. 

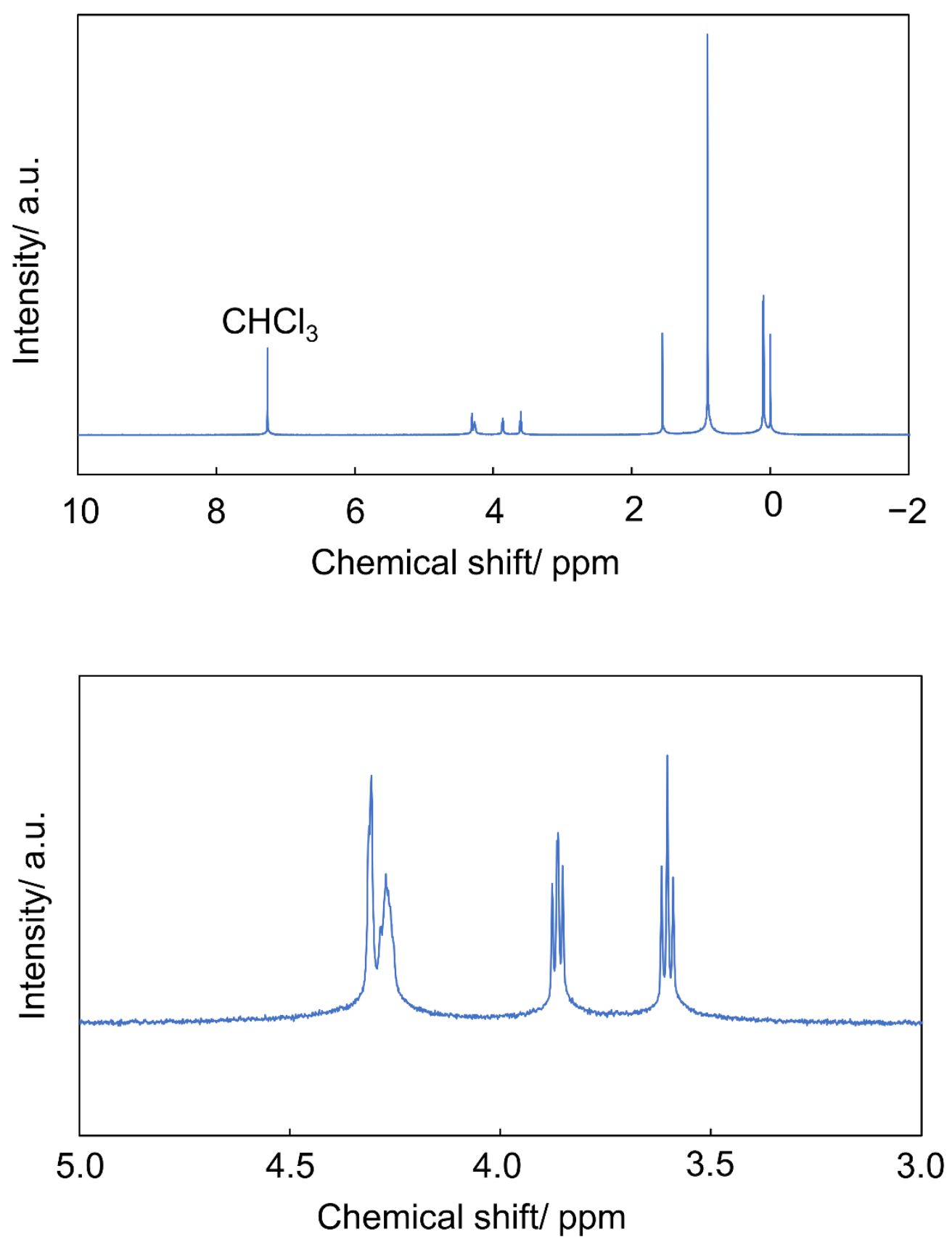

Figure S1. ${ }^{1} \mathrm{H}$ NMR spectrum of 2 . Top: full scale, Bottom: enlarged view. 

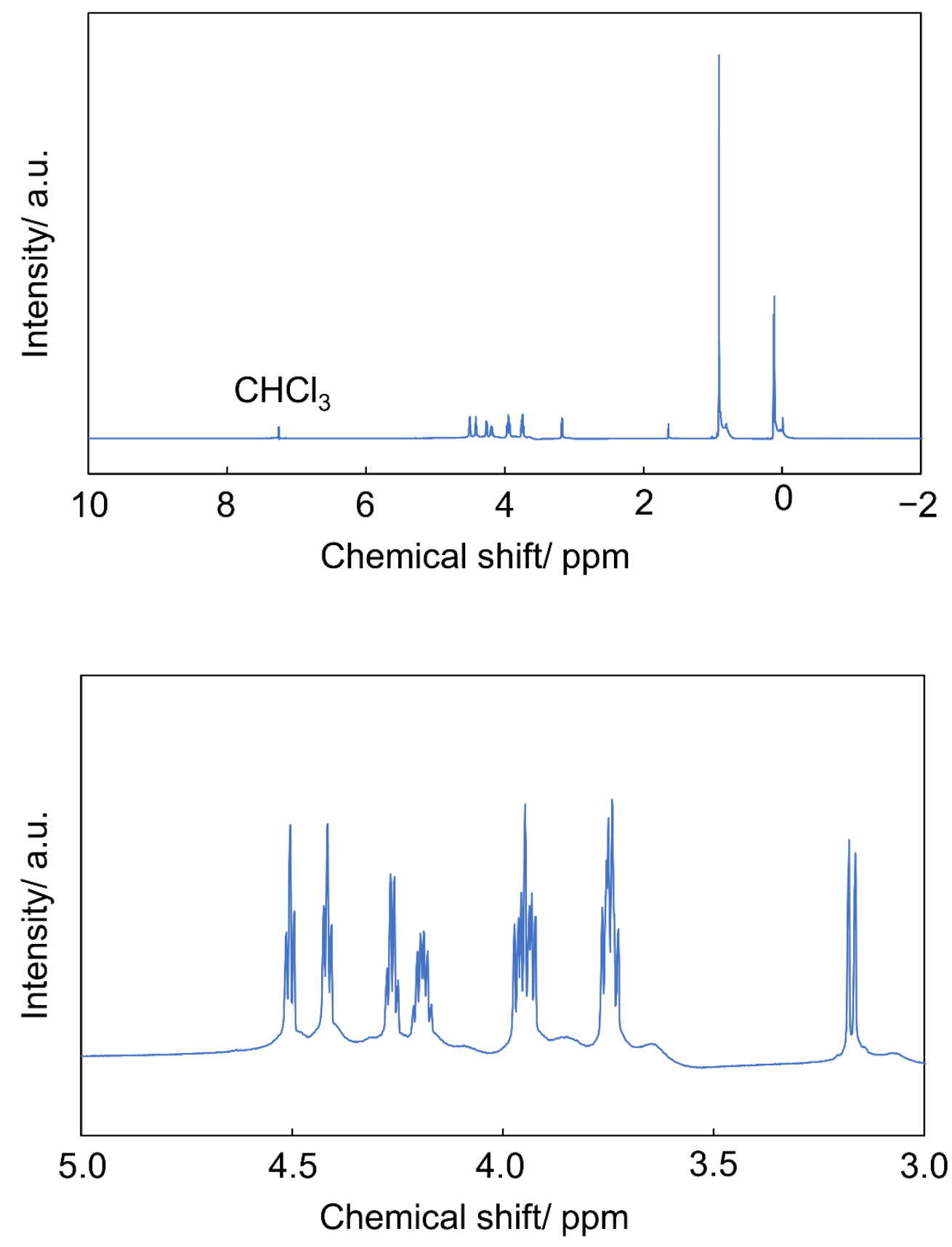

Figure S2. ${ }^{1} \mathrm{H}$ NMR spectrum of 3. Top: full scale, Bottom: enlarged view. 

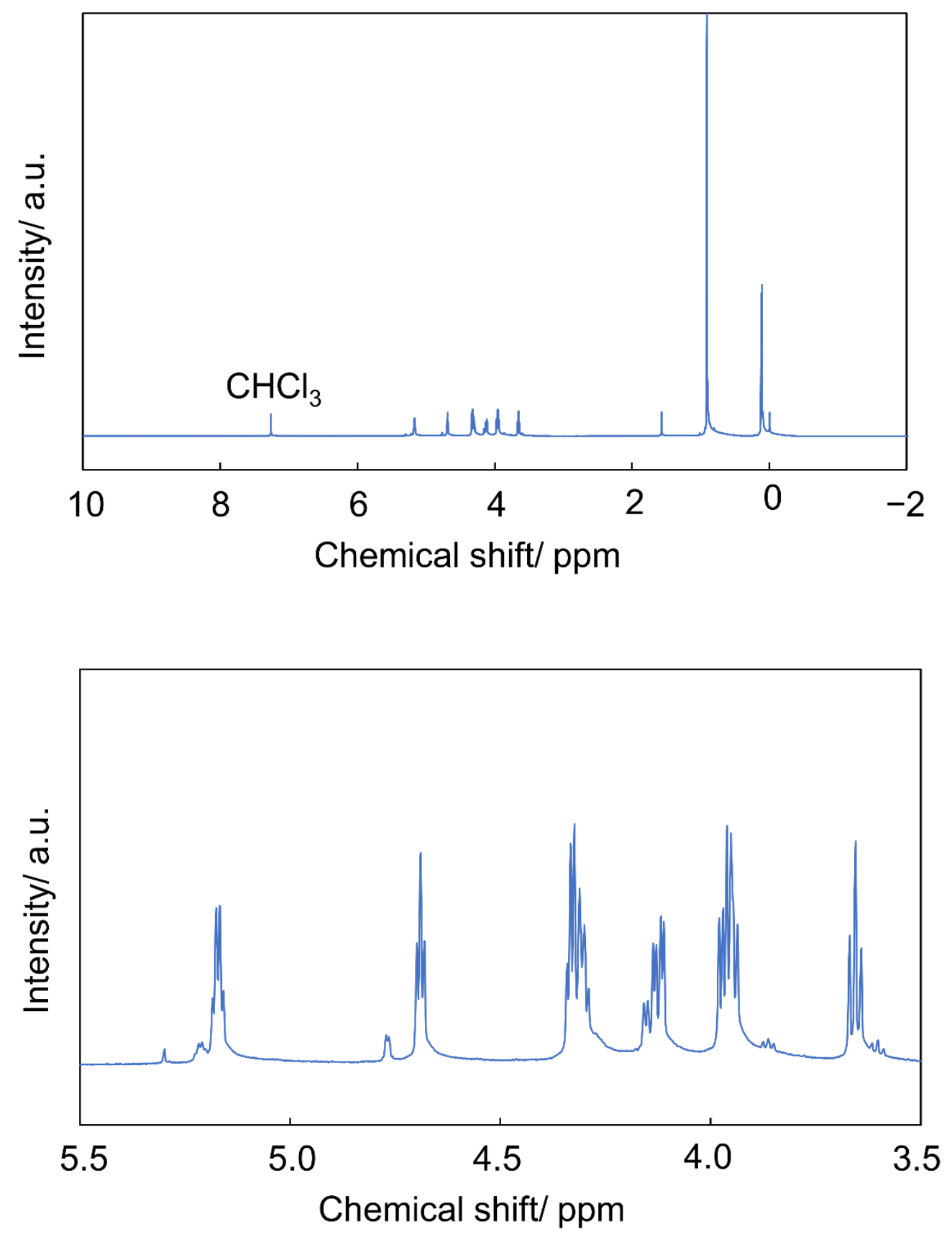

Figure S3. ${ }^{1}$ H NMR spectrum of 4 . Top: full scale, Bottom: enlarged view. 

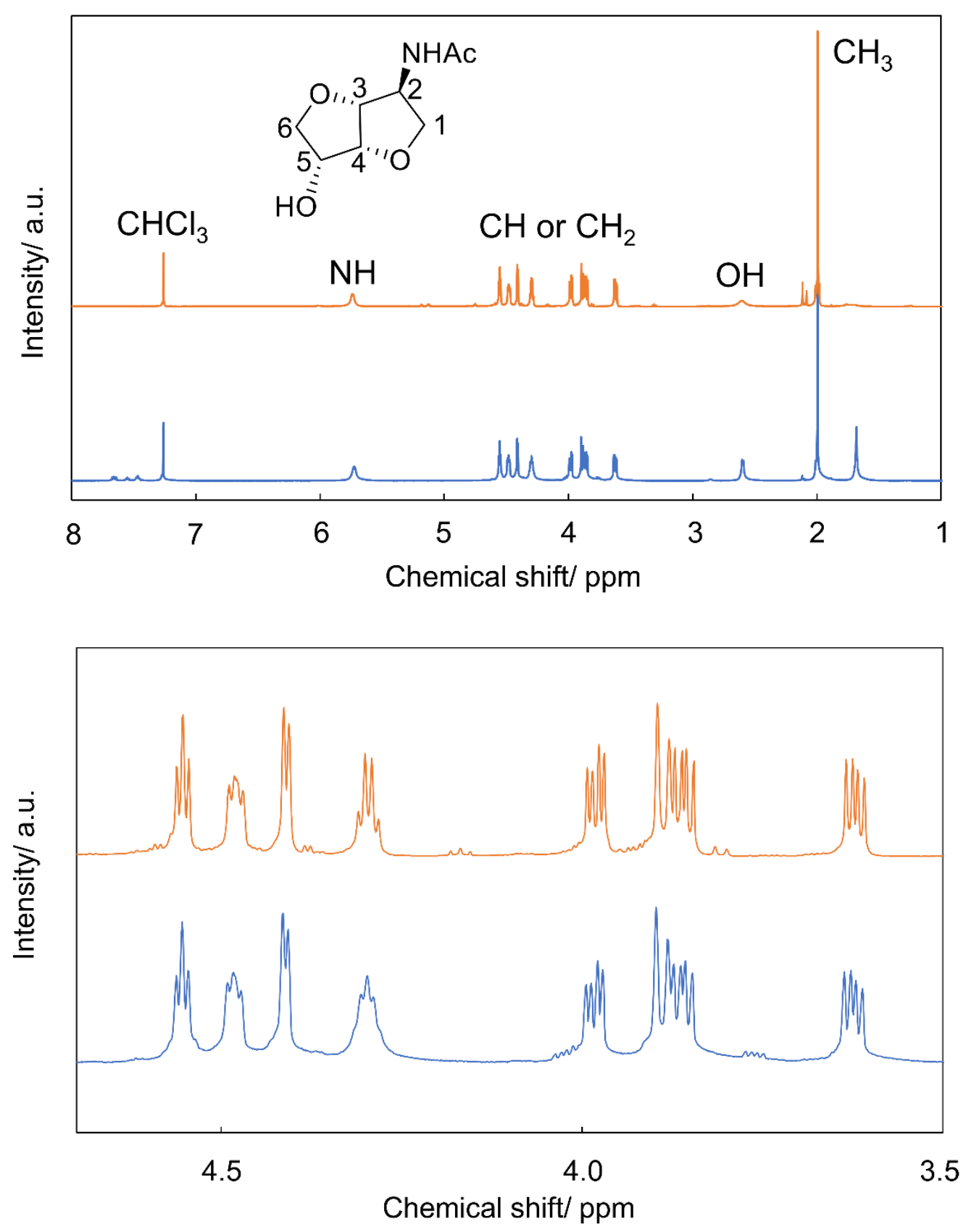

Figure S4. ${ }^{1} \mathrm{H}$ NMR spectra of the standard ADI (blue) synthesized from isomannide (Scheme S1) and that produced from ADS (orange). Top: full scale, Bottom: enlarged view. In the spectrum of standard ADI, the signal at $1.6 \mathrm{ppm}$ is assigned to $\mathrm{H}_{2} \mathrm{O}$ and the signals at 7.5-7.7 ppm are assigned to residual $\mathrm{Ph}_{3} \mathrm{P}=\mathrm{O}$. Peaks at $2.1 \mathrm{ppm}$ in the orange spectrum were derived from impurities that appeared in the HPLC chromatogram as a shoulder peak next to the peak of ADI. Peaks at 4.3 ppm in the two spectra have different splitting patterns. The peak is derived from $\mathrm{C} 5-H$ of ADI. For the standard ADI (blue), the peak splitted into a complicated pattern (theoretically dddd, coupling with $\mathrm{C} 4-\mathrm{H}, \mathrm{C} 5-\mathrm{OH}$ and two different C6- $H$ ). However, ADI produced from ADS (orange) gave a quartet-like pattern which is actually assumed to be ddd. In this measurement, $\mathrm{OH}$ peaks were broadened due to fast proton exchange as seen for $\mathrm{C} 5-\mathrm{OH}$ (2.59 ppm) and water (1.7 ppm, very small broad peak), and therefore ${ }^{1} \mathrm{H}-{ }^{1} \mathrm{H}$ J-coupling with $\mathrm{C} 5-\mathrm{O} H$ disappeared. 


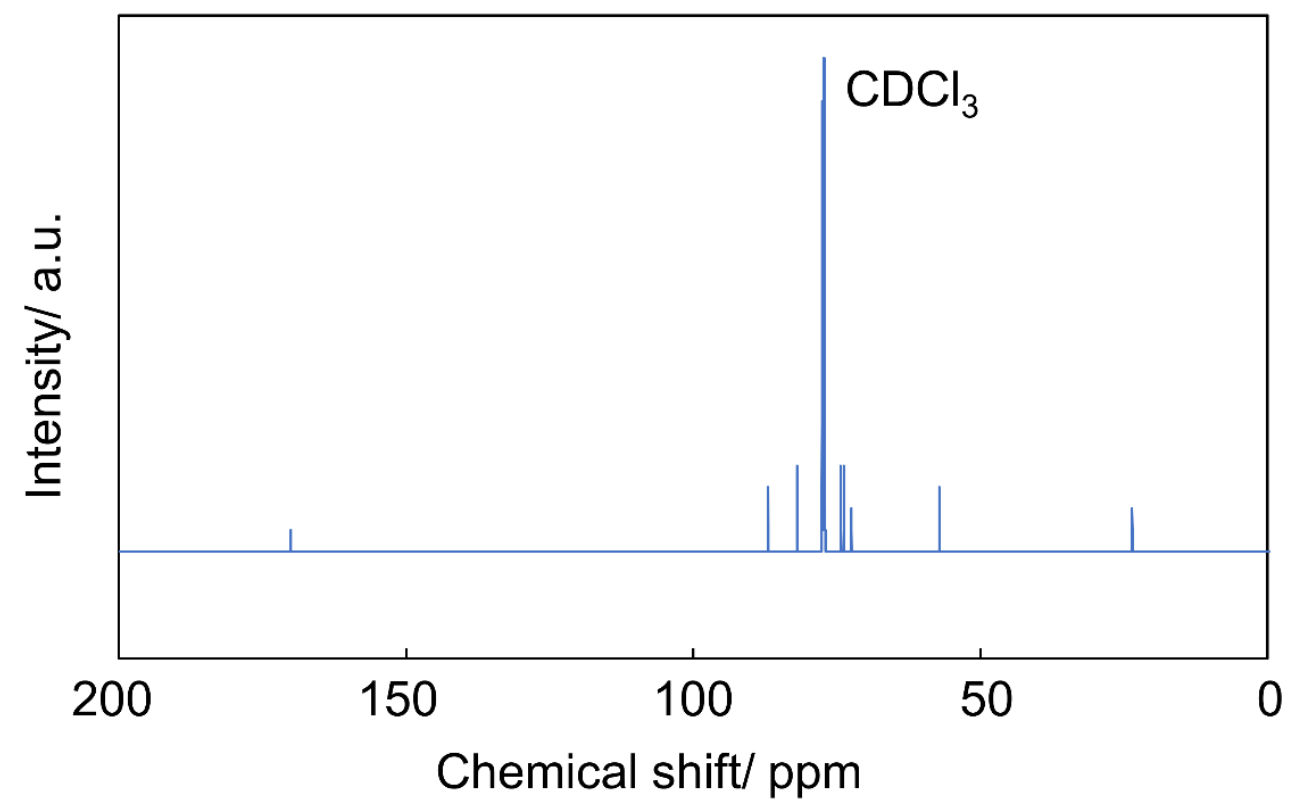

Figure S5. ${ }^{13} \mathrm{C}$ NMR spectrum of ADI.

We have measured ${ }^{13} \mathrm{C}$ NMR spectra of both standard ADI and ADI produced from ADS. The peak positions were the identical. This figure shows the spectrum of ADI produced from ADS.

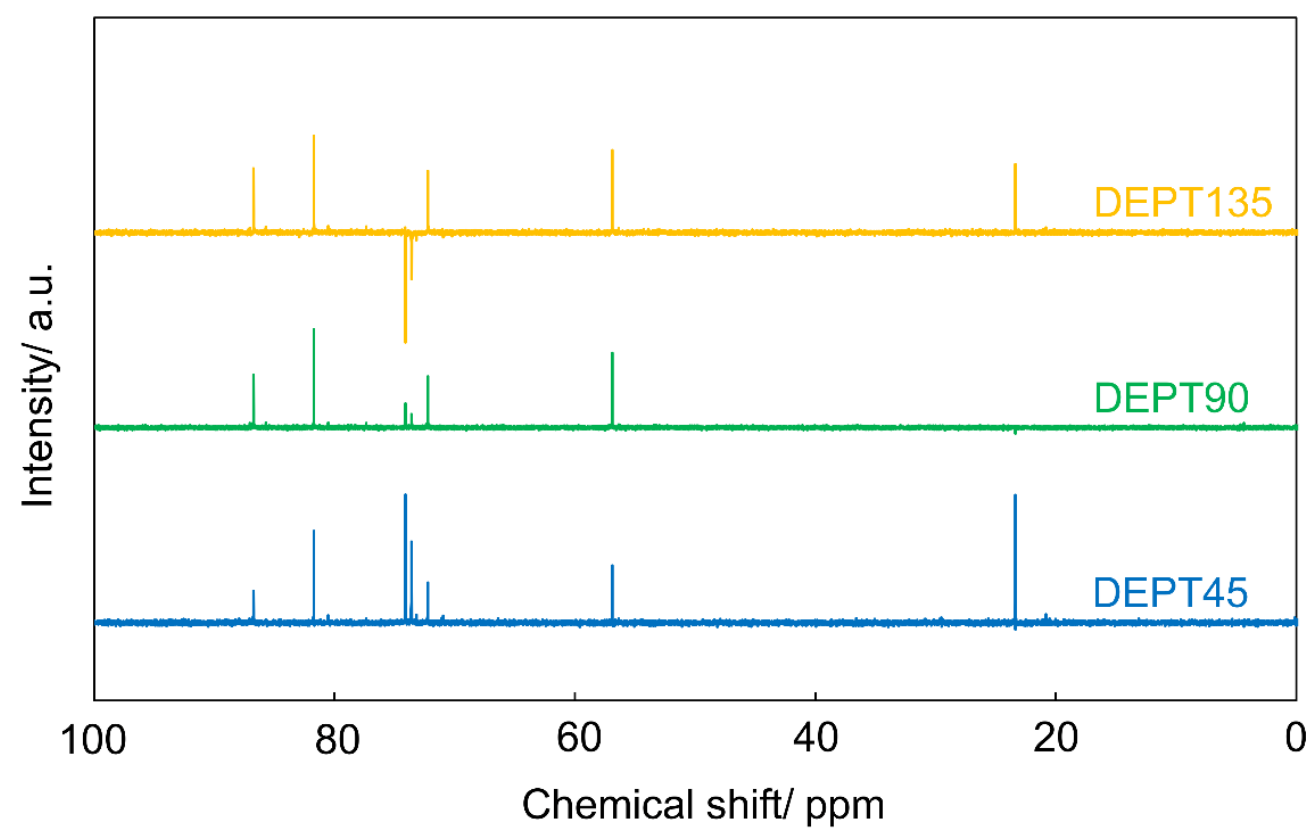

Figure S6. DEPT spectra of ADI. 


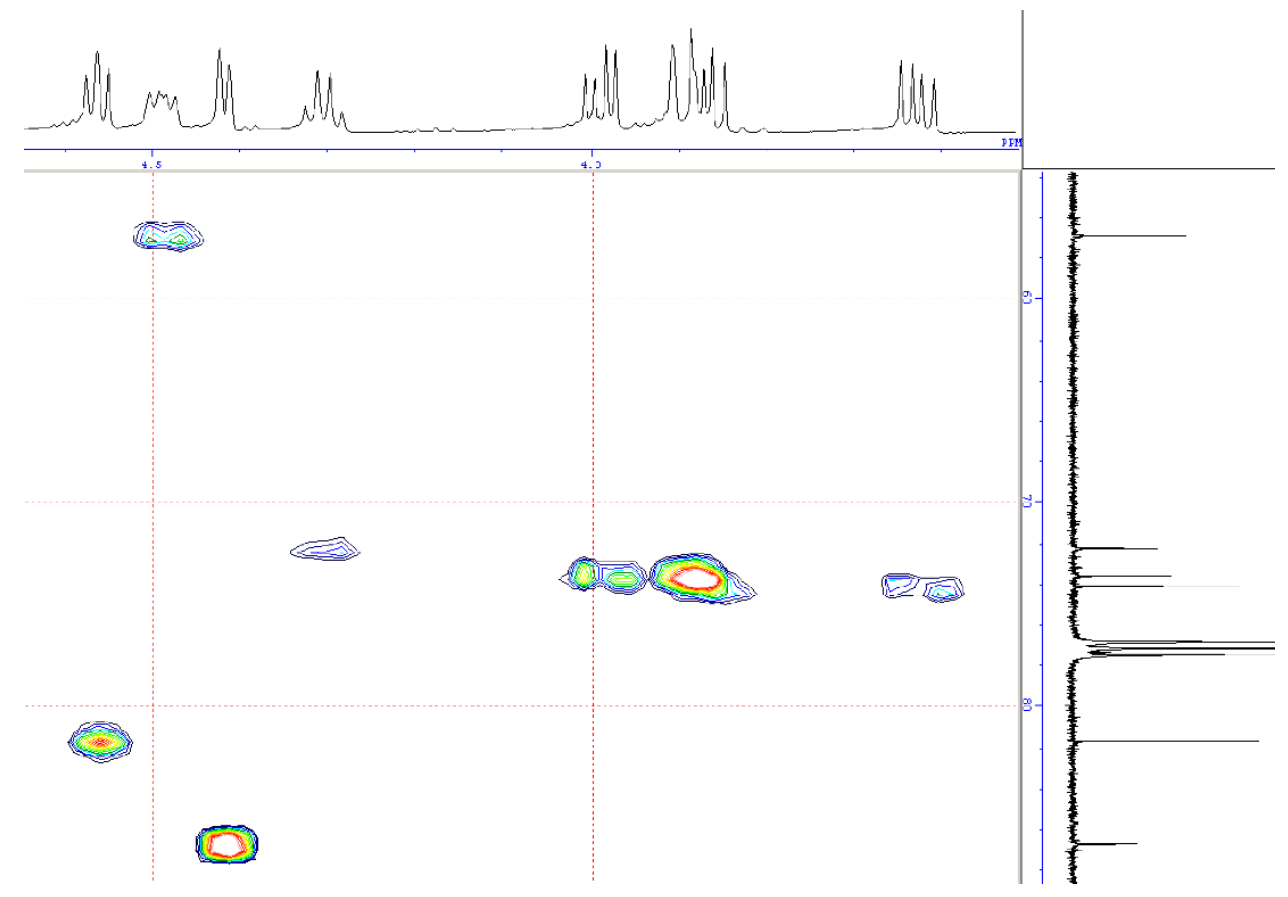

Figure S7. ${ }^{13} \mathrm{C}-{ }^{1} \mathrm{H}$ HMQC spectrum (horizontal axis: ${ }^{1} \mathrm{H}$, vertical axis: ${ }^{13} \mathrm{C}$ ) of ADI.

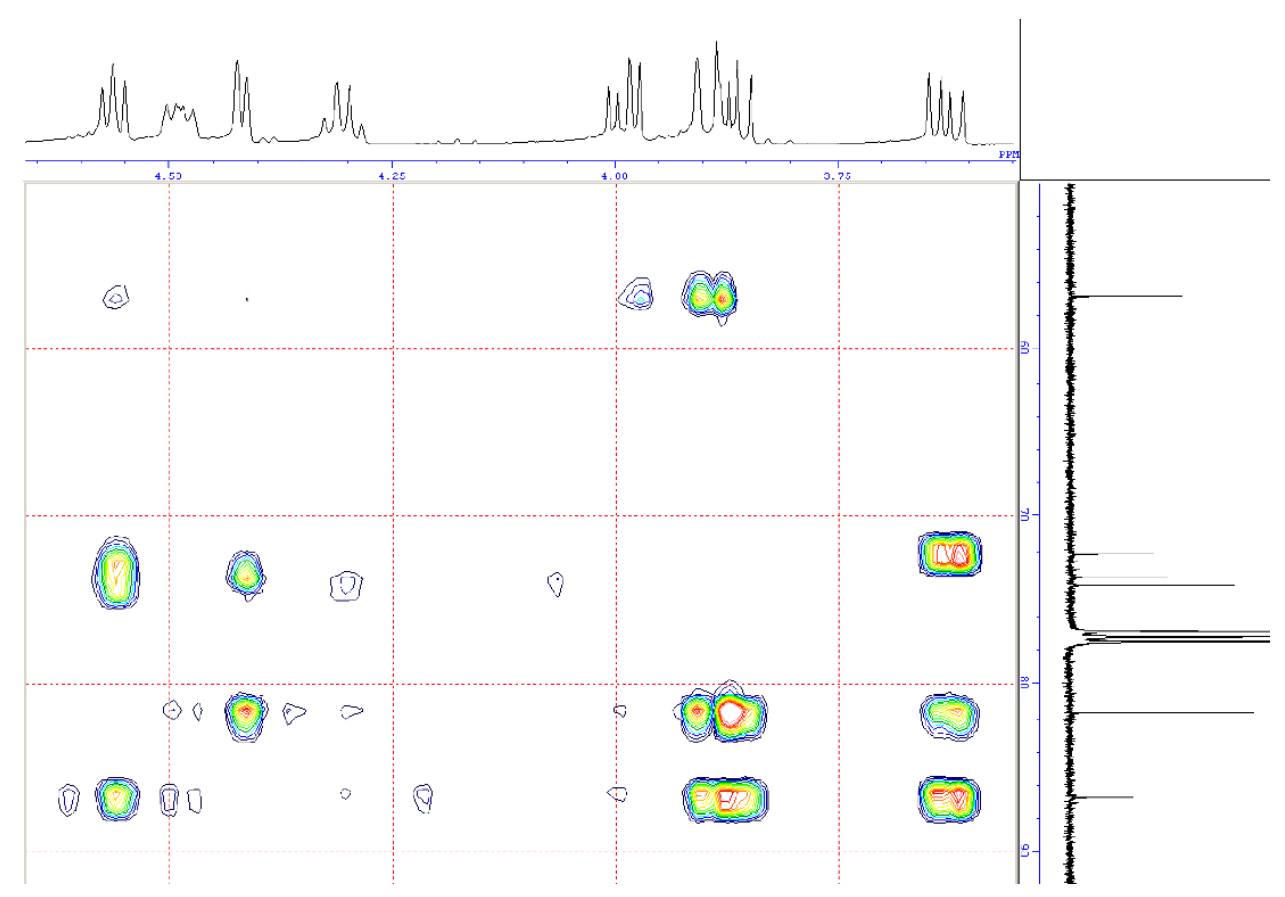

Figure S8. ${ }^{13} \mathrm{C}-{ }^{1} \mathrm{H}$ HMBC spectrum (horizontal axis: ${ }^{1} \mathrm{H}$, vertical axis: ${ }^{13} \mathrm{C}$ ) of ADI. 


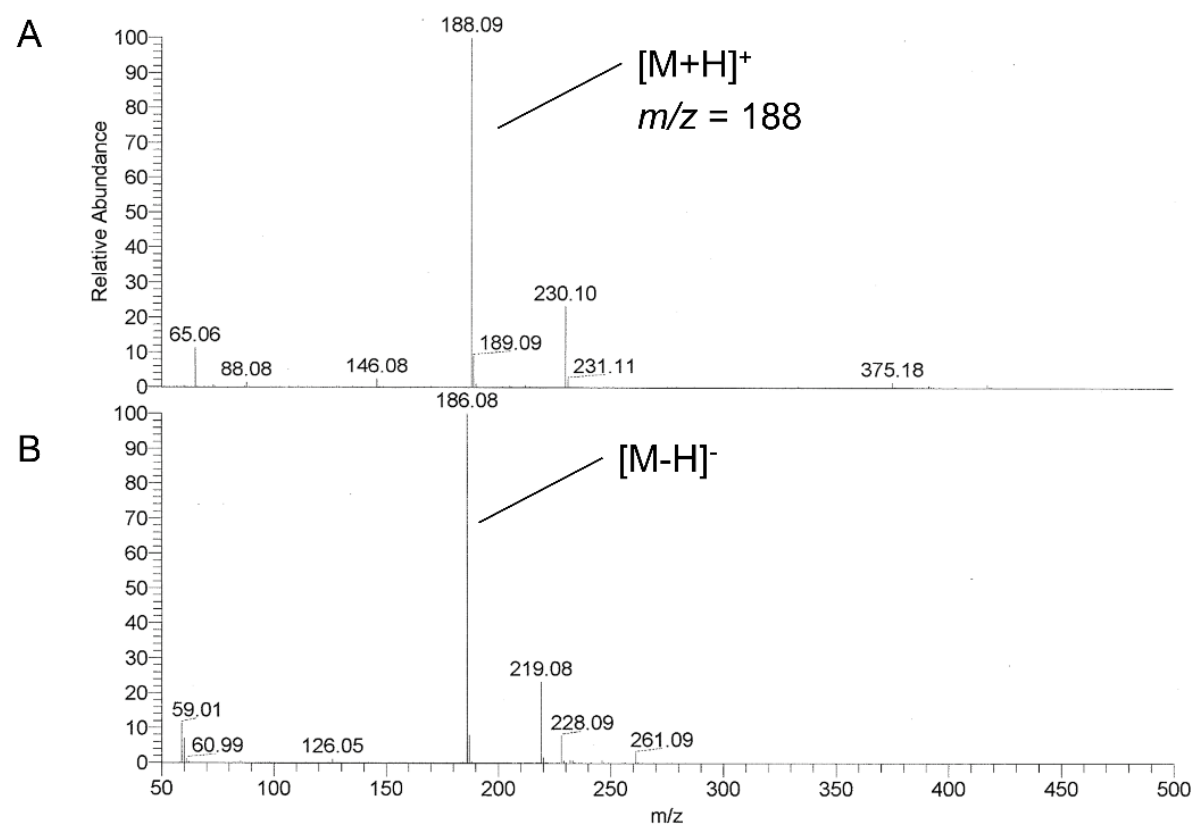

Figure S9. LR-APCI positive (A) and negative (B) mass spectra of ADI produced from ADS.

A

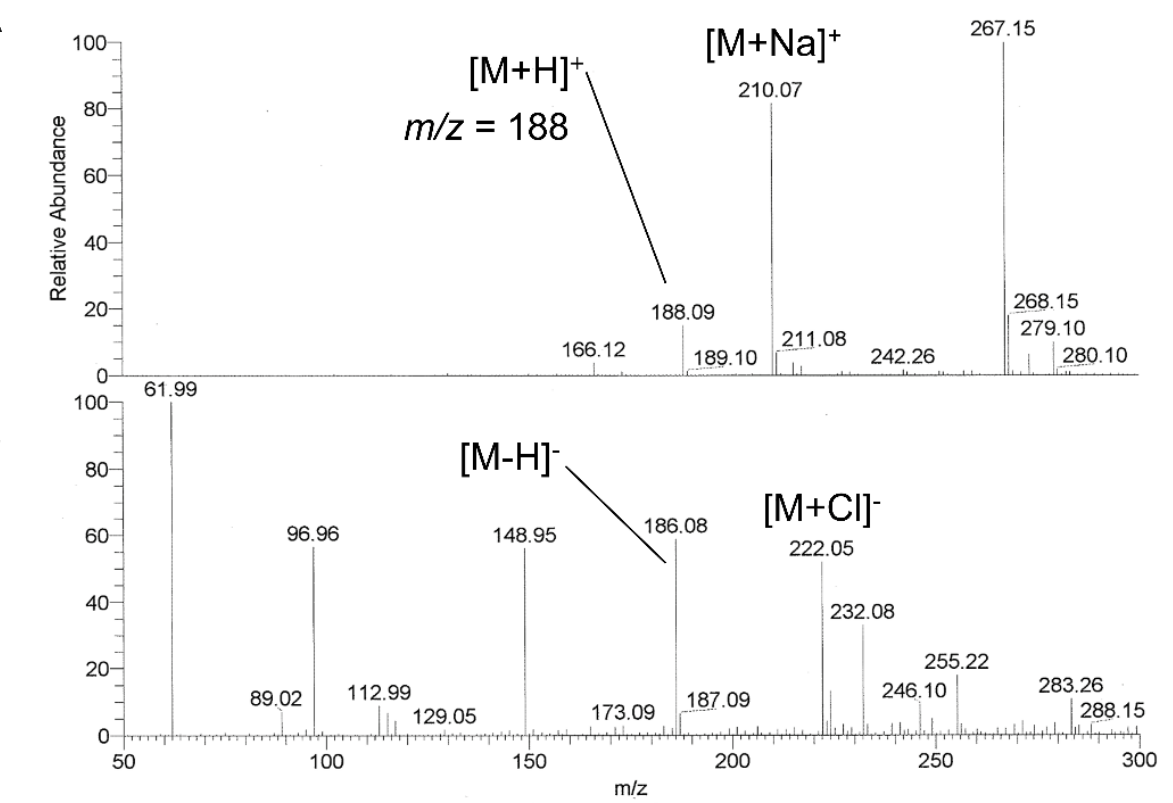

Figure S10. LR-ESI positive (A) and negative (B) mass spectra of standard ADI synthesized from isomannide (Scheme S1). 
Table S1. Dehydration cyclization of ADS and sorbitol by $\mathrm{H}_{2} \mathrm{SO}_{4}{ }^{a}$

\begin{tabular}{llllll}
\hline Entry & Substrate & Conv. & Yield of product $/ \%$ & & \\
\cline { 3 - 6 } & & & & & \\
\cline { 3 - 6 } & & & Mono-anhydro sugar alcohols & Di-anhydro sugar alcohol & Others \\
\hline S1 & ADS & 20 & 11 & $<0.1$ & 9 \\
S2 & Sorbitol & $>99$ & 3 & 72 & 24
\end{tabular}

${ }^{a}$ Reaction conditions: sugar alcohol $1.00 \mathrm{mmol}, \mathrm{H}_{2} \mathrm{SO}_{4} 0.64 \mathrm{mg}(6.5 \mu \mathrm{mol}, \mathrm{S} / \mathrm{C}=150)$, no solvent, $130{ }^{\circ} \mathrm{C}, 3 \mathrm{~h}, 100 \mathrm{kPa}$. 